\title{
Evaluación de los parámetros físicos y químicos de las briquetas obtenidas con la maquina briquetadora construida en FAREM- Estelí
}

\author{
Juan David López Rivera ${ }^{1}$ \\ Carlos Eduardo Cajina Valdivia ${ }^{2}$ \\ Jorge Ulises Ramírez Camas ${ }^{3}$ \\ Edwin Antonio Reyes Aguilera ${ }^{4}$ \\ Noé Rodolfo Olivas Reyes ${ }^{5}$ \\ Lidamar de los Angeles Molina Cruz ${ }^{6}$
}

\section{RESUMEN}

El propósito fundamental de esta investigación es evaluar los parámetros físicos y químicos de las briquetas elaboradas en Farem-Estelí. Es una investigación cuantitativa según su enfoque, con un nivel de profundidad según su tipología: exploratoria, descriptiva. Se elaboraron siete briquetas de diferentes sustratos forestales. Los instrumentos utilizados fueron: La evaluación de eficiencia que se determinó mediante la prueba de ebullición de agua (WBT por sus siglas en inglés) versión 4.2.2., se midieron las emisiones de monóxido de carbono ( $\mathrm{CO}$ ) y se calculó el poder calorífico de los diferentes tipos de briquetas. Los resultados obtenidos muestran que estas briquetas emiten menos emisiones de gases contaminantes al ambiente que la leña, la eficiencia térmica fue de aproximadamente un $92 \%$ en tres de las briquetas evaluadas, la leña obtuvo una eficiencia entre 45 y $63 \%$. Se concluye que la elaboración de las briquetas fue exitosa con una excelente compactación, la briqueta de Granza 50\% + Aserrín fino 25\% + Papel 12.5\% + flor de pino $12.5 \%$ es la mejor opción según la evaluación siendo esta la que emite menores cantidades de CO que son casi nulas obteniendo una eficiencia térmica de $92 \%$ puesto que su consumo especifico es bajo con tan solo 143 gramos por litro de agua.

Palabras claves: Briquetas, monóxido de carbono, eficiencia térmica.

Recibido: 09 de diciembre de 2015

Aceptado: 29 de enero de 2016

1 UNAN-Managua/FAREM-Estelí. Correo Electrónico: lopezjuan_13@yahoo.es 2 UNAN-Managua/FAREM-Estelí. Correo Electrónico: carlos.valdivia06@gmail.com 3 UNAN-Managua/FAREM-Estelí. Correo Electrónico: jorgeulices@yahoo.com 4 UNAN-Managua/FAREM-Estelí. Correo Electrónico: edwinra11@yahoo.es 5 UNAN-Managua/FAREM-Estelí. Correo Electrónico: noeolivasreyes@yahoo.es 6 UNAN-Managua/FAREM-Estelí. Correo Electrónico: lidamar.molina@gmail.com 


\section{Evaluation of physical and chemical parameters of the briquettes obtained with the briquetting machine built in FAREM - Estelí}

\section{SUMMARY}

The main purpose of this research is to evaluate the physical and chemical parameters of the briquettes produced in Farem - Estelí. It is a quantitative, exploratory and descriptive research. Seven different briquettes of forest substrates were produced. The instruments used were: The efficiency evaluation was determined by water boiling test (WBT for its acronym in English) version 4.2.2, emissions of carbon monoxide (CO) were measured, and the calorific power of the different types of briquette was calculate too. The results show that the briquettes emit fewer gas emissions to the environment than the firewood, the thermal efficiency was approximately $92 \%$ in three of the tested briquettes, and the firewood got efficiency between 45 and $63 \%$. It is concluded that the development of the briquettes was successful with an excellent compaction, briquette of Granza 50\% + fine sawdust $25 \%$ + paper $12.5 \%+$ paper of pine flower $12.5 \%$ is the best option according to the research, being this the one that emits smaller amounts of CO that are almost nil, this obtained a thermal efficiency of $92 \%$ since its specific consumption is low with only 143 grams per liter of water.

Keywords: Briquettes, carbon monoxide, thermal efficiency 


\section{INTRODUCCIÓN}

En el mundo un promedio de tres mil millones de personas utilizan leña como combustible para fines de cocina y calefacción, de los cuales dos mil millones de personas se encuentran en áreas rurales y suburbanas (SHE, 2004). En América Latina y el Caribe cada año se consume 254 millones de $\mathrm{m}^{3}$ de leña (FAO, 1999). De manera que los más afectados resultan ser las personas que habitan en zonas rurales principalmente las mujeres y niños que ahora tienen que recorrer mayor distancia para recolectar leña, generando problemas sociales y ambientales (FAO, 2005).

Desde el año 1992 hasta el año 2003 Legacy Foundation y sus socios han realizado estudios sobre el uso de la tecnología de briquetas en países asiáticos, africanos y latinoamericanos (Perú, Nicaragua y Haití) en ambientes rurales y suburbanos. Por la escasez y la poca accesibilidad la tecnología de las briquetas está orientada a sustituir el uso de la leña. En los lugares donde se desarrolló la tecnología de las briquetas se ha reducido el consumo de leña y se ha dado mayor aprovechamiento a los residuos que generan las actividades agrícolas y de la industria maderera (Legacy Foundation, 2003).

La deforestación ha causado un impacto negativo a nivel mundial y Nicaragua no es la excepción, según MAGFOR alrededor de 70000 hectáreas son taladas cada año, lo que trae consigo una elevada cantidad de residuos forestales, los cuales generan contaminación, vuelven propensa a la localidad a incendios, atraen plagas indeseables entre otros efectos perjudiciales para la población.

Los residuos forestales son inevitables debido a la naturaleza destructiva del hombre, que utilizan los bosques para generar energía en forma de calor y utilizarla en hornos o cocinas de leña entre otra aplicaciones, lo cual no afecta solo a la contaminación ambiental sino también a la salud de las personas que las usan, ya que el humo emitido por estos productos genera enfermedades pulmonares obstructivas graves que pueden llegar a provocar la muerte'

Pero hay una alternativa ecológica que puede tratar estos residuos de tal manera que se aprovechen las cualidades como lo son su excelente capacidad de combustión para generar calor a bajos niveles de contaminación y con valor económico factible, mediante la compactación de residuos forestales las denominadas briquetas ${ }^{2}$.

La mayor limitante del estudio fue no contar con suficientes materiales bibliográficos tradicionales que nos revelen datos y experiencias de la aplicación de la tecnología de las briquetas. Por esta razón se utilizan manuales y materiales digitales como tecnología nueva y la experiencia adquirida de Legacy Foundation, para entender mejor el tema de briquetas. En el primer semestre del 2015 en FAREM ESTELI se construyó una maquina briquetadora funcional de la cual se obtuvieron briquetas con un excelente nivel de compactación las cuales en teoría, se considera que son de gran beneficio tanto económico como ambiental pero que no fueron evaluados pertinentemente para realizar un estudio completo.

Una briqueta es un biocombustible para generar calor utilizados en estufas, chimeneas, hornos y calderas, normalmente en forma cilíndrica o cuadrada y es un sustituto de la leña con muchas ventajas. Generalmente hechas con materia residual, como cascarilla de arroz, aserrín, papel, cartón, o cualquier otro residuo ${ }^{3}$.

Actualmente el manejo de residuos en el sector maderero ha tomado mayor interés, con el fin de hacer más productivas las empresas de este rubro.

\footnotetext{
1 Morini, S. (2011). Perdidas y Desperdicios de Recursos Forestales en el Mundo.

2 Kollman, F. (2007). Tecnologia de la madera y sus aplicaciones.

3 Barrera, J. (2008). Briquetas, la mejor forma de reutilizar los residuos forestales
} 
Variados son los productos que se generan a partir de los desechos maderables pero aún queda gran cantidad de residuos de madera, por lo que las empresas se ven obligadas a desecharlos en los ríos o incinerarlos al aire libre causando problemas ambientales ${ }^{4}$.

Por tanto es conveniente realizar evaluaciones de las emisiones de monóxido de carbono que nos permiten determinar el nivel de contaminación a la capa de ozono que generan las briquetas de diferentes sustratos forestales, así mismo la capacidad de combustión que es algo fundamental si se pretende emplear para la quema y generar calor, pues si no tiene una buena capacidad de combustión es insostenible. Este documento permite ser utilizado como herramienta de capacitación y el uso de las briquetas sea extendido a personas y entidades que lo requieran. Se generó un documento de recetas que nos permitió determinar cuál de las mezclas es más factible como alternativa al uso de leña tradicional y aprovechar los residuos de actividades ganaderas, agrícolas y plantas de procesamiento de madera.

\section{MATERIALES Y MPETODOS}

La investigación pretende fundamentalmente describir los pasos que se tomaron en cuenta en la evaluación de los parámetros físicos y químicos de las briquetas obtenidas del prototipo de maquina briquetadora elaborado en FAREM-ESTELI.

\section{Ubicación del área de estudio}

Esta investigación de llevo a cabo en la Facultad Regional Multidisciplinaria (FAREM, Estelí).

Tipo de estudio

La presente investigación es cuantitativa según su enfoque, con un nivel de profundidad según su tipología: exploratoria, descriptiva.

4 Ortiz, L. (2003). La Biomasa como fuente de energía.

\section{Fases de la investigación}

La presente investigación se constituye de tres etapas fundamentales para la evaluación de los parámetros físicos y químicos de las briquetas las cuales son las siguientes: etapa de elaboración de las briquetas, etapa de pre-evaluación y finalizando con la etapa de evaluación.

\subsubsection{Etapa de elaboración de las briquetas.}

Aprovechando la funcionalidad del prototipo de maquina briquetadora para la compactación de diferentes sustratos, se fabricaron diferentes tipos de briquetas, con diferentes proporciones seleccionadas de manera experimental pues no se cuenta con un manual que indique la cantidad de proporciones de sustratos para una elaboración óptima.

Los residuos con los que se elaboraron las briquetas son los siguientes: Aserrín fino (Obtenido de carpintería privada), Colocho de aserrín (obtenido de carpintería privada) Papel (proporcionado por la fotocopiadora de FAREM-ESTELI, realiza la función de aglutinante), Flor de pino (extraída de área protegida el Tisey), Zacate seco: (de la limpieza que se realiza en FAREMESTELI), Ramitas (recolectadas del árboles en FAREM-ESTELI)

Se elaboraron briquetas de manera independiente, con diferentes proporciones de mezclas para su posterior evaluación. Los pasos para su fabricación fueron los siguientes.

\section{Recolección y acumulación de residuos: para} fabricar las briquetas se recolectaron y acumularon los diferentes residuos forestales de empresas privadas para las cuales es una pérdida de espacio y tiempo el tratamiento de los mismos.

2. Trituración o picado de los residuos: un paso importante en la fabricación de las briquetas, este se 
realiza de acuerdo a los materiales de elaboración y a sus proporciones. Algunos pueden tener un mayor picado o menor en dependencia de la capacidad de amarre del material, lo que quiere decir que por

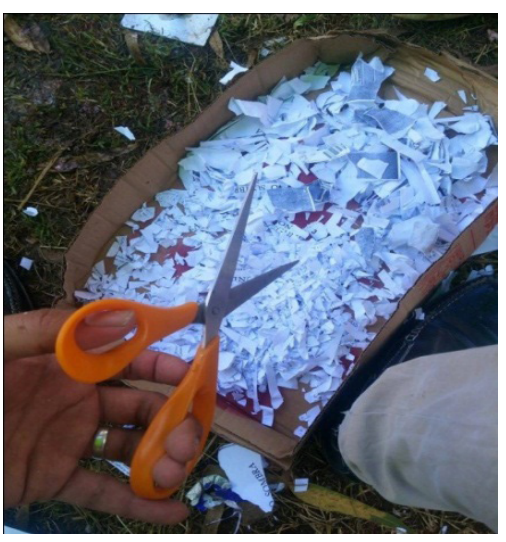
ejemplo al elaborar

una briqueta de aserrín este no posee la capacidad de amarre o pegado para formar una briqueta por lo cual se añade papel triturado como aglutinante en cierta proporción. Hay varias maneras de triturar o picar los residuos, con maquina especializada para triturar eléctrica con la cual no se contó para el estudio, con licuadora o realizándolo de manera manual.

\section{Realizar las proporciones de residuos para la} posterior mezcla: es fundamental tomar en cuenta el peso de cada sustrato, y la cantidad que se agregara a la mezcla, se realizó diferentes mezclas pretendiendo realizar una comparativa para determinar cuál porcentaje de residuo es óptimo para la elaboración de la briqueta.

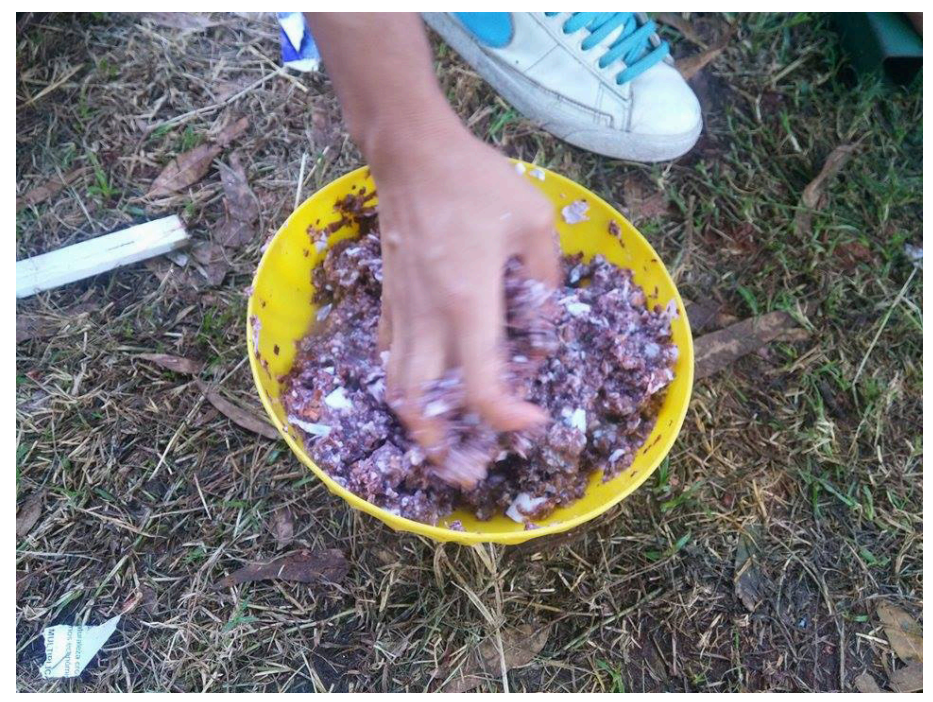

4. Mezclar con agua o aglutinante: en un recipiente plástico para todas las briquetas se empleó $500 \mathrm{ml}$ de agua para realizar una mejor mezcla de los residuos y se utilizó cierto porcentaje (el cual se tratara posteriormente) de papel en algunas de las briquetas que sirvió como aglutinante para contribuir al amarre de los residuos. Esta mezcla se revolvió por 10 minutos para realizar un mezclado uniforme y una mejor adhesión entre los residuos.

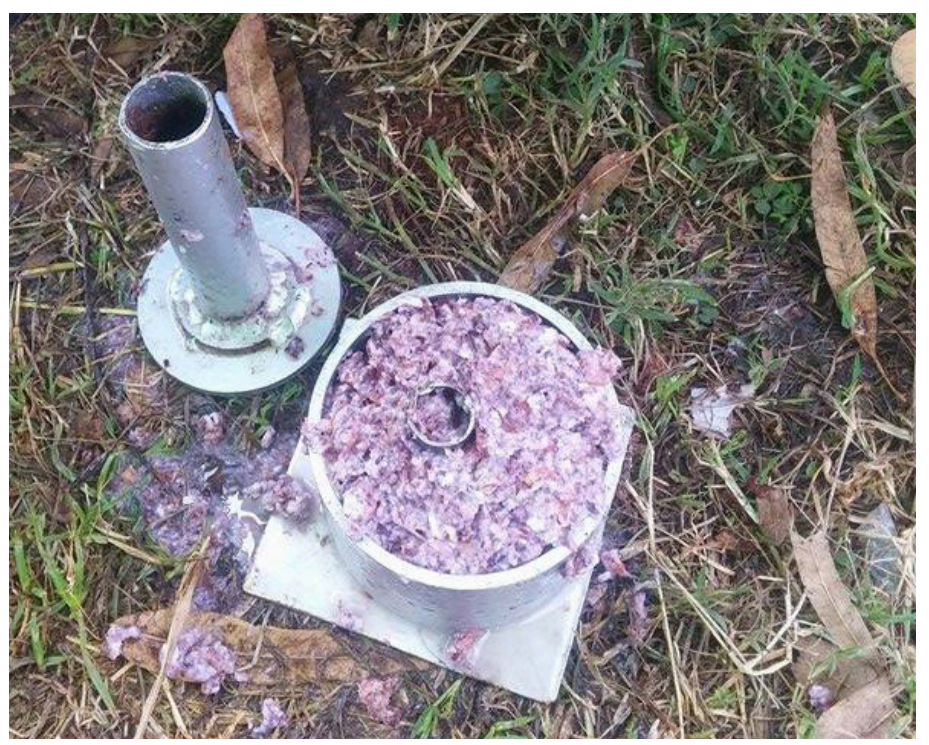

5. Verter la mezcla en el molde: se vacía la mezcla homogénea en el recipiente movible del prototipo de maquina briquetadora el cual le dará la forma circula con un orificio al centro lo cual permite la entrada de oxígeno a la briqueta para su combustión.

\section{Realizar la compactación de la mezcla: se realiza un} prensado con el prototipo de maquina briquetadora ejerciendo un presión de dos toneladas para extraer de manera eficiente el líquido de la briqueta $\mathrm{y}$ realizar una compactación solida de la mezcla de los diferentes sustratos.

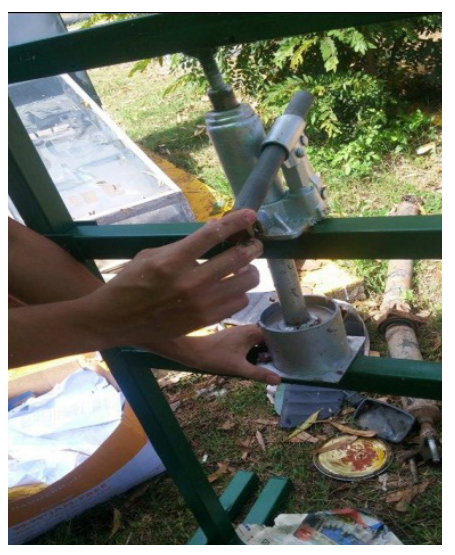


7. Secado de las briquetas: hay varios métodos de secado entre estos, con horno eléctrico, secador solar, o por secado natural, se optó por el secado natural exponiendo las briquetas a radiación solar directa para eliminar la humedad de las mismas y la briqueta tuviera una compactación correcta, el periodo de tiempo que se le dio a las briquetas con aire constante y radiación solar directa fue de 3 días.

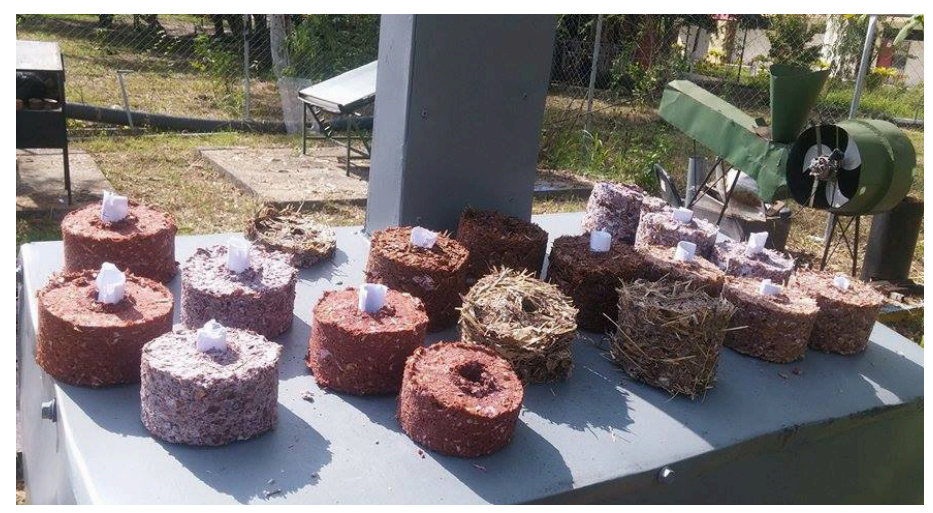

\section{Etapa de pre-evaluación de las briquetas}

Esta etapa se tomó en cuenta para los resultados finales, en esta se fabricaron briquetas de 300 gramos cada una para tener varias muestras y tomar en cuenta aspectos importantes para seleccionar las briquetas adecuadas y no hacer una evaluación en vano. Los dos aspectos importantes que se medirán son los siguientes:

\section{Prueba física}

Las briquetas tienen una forma circular con un orificio en medio que están establecidos por el molde, en esta prueba se quiere tratar que las briquetas al ser manipuladas durante su fabricación, transporte y secado conserven su estructura. Esto se calificó de la siguiente manera:

- Excelente: cuando la briqueta a la hora de su manipulación la estructura es lo suficientemente estable y en su secado tiene que estar de manera dura.

- Regular: cuando la briqueta a la hora de su manipulación la estructura no es tan estable y en su secado sea duro pero se desmorone y rompa con mayor facilidad.

- Malo: la estructura no fue estable, es decir a la hora de su manipulación la estructura tienda a volver a su estado original, y cuando este seco se desmorone y rompa fácilmente.

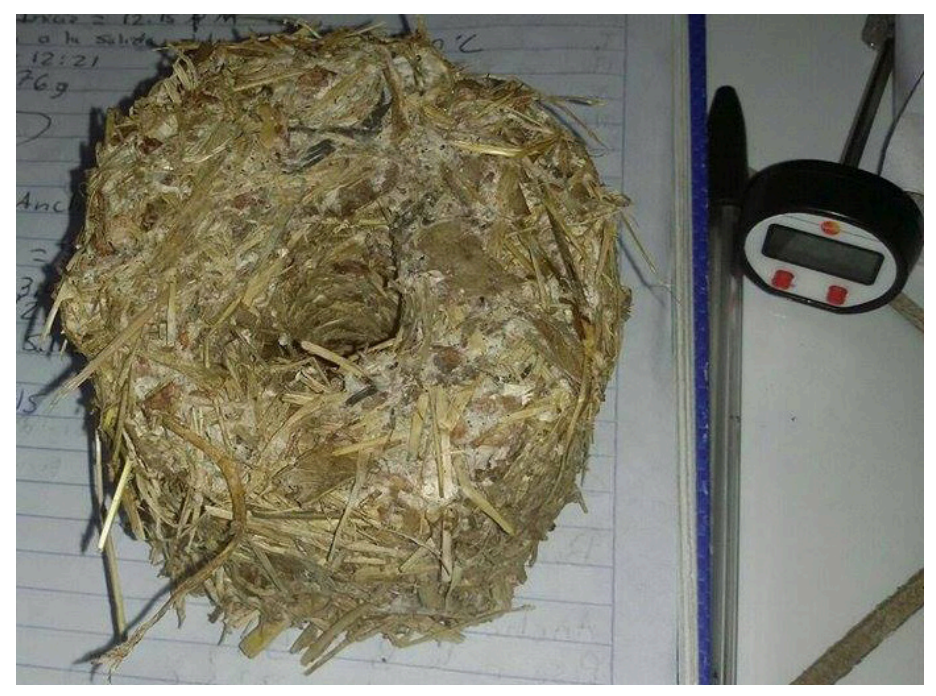

\section{Prueba de encendido}

El encendido de la briqueta depende de gran manera de la ventilación en la que se realice la prueba para efectos de prueba y no alterar se realizó en el laboratorio con poca ventilación. Esta prueba consiste en medir la facilidad que tienen las briquetas en encender para lo cual se utilizó una cama de madera u ocote igual para todas las briquetas y se calificó de la siguiente manera:

- Enciende: cuando al quemarse la cama las briquetas quedan encendidas por un tiempo considerable hasta consumirse.

- No enciende: cuando al quemarse la cama las briquetas no enciendan o enciendan y se apaguen sin quemarse por completo.

Para el proceso de evaluación se seleccionaran el tipo de briquetas que en su prueba física sea excelente y naturalmente su encendido fácil, se tomaron en cuenta las proporciones de los sustratos utilizados en las briquetas y la cantidad de papel que contienen si es que contienen. 


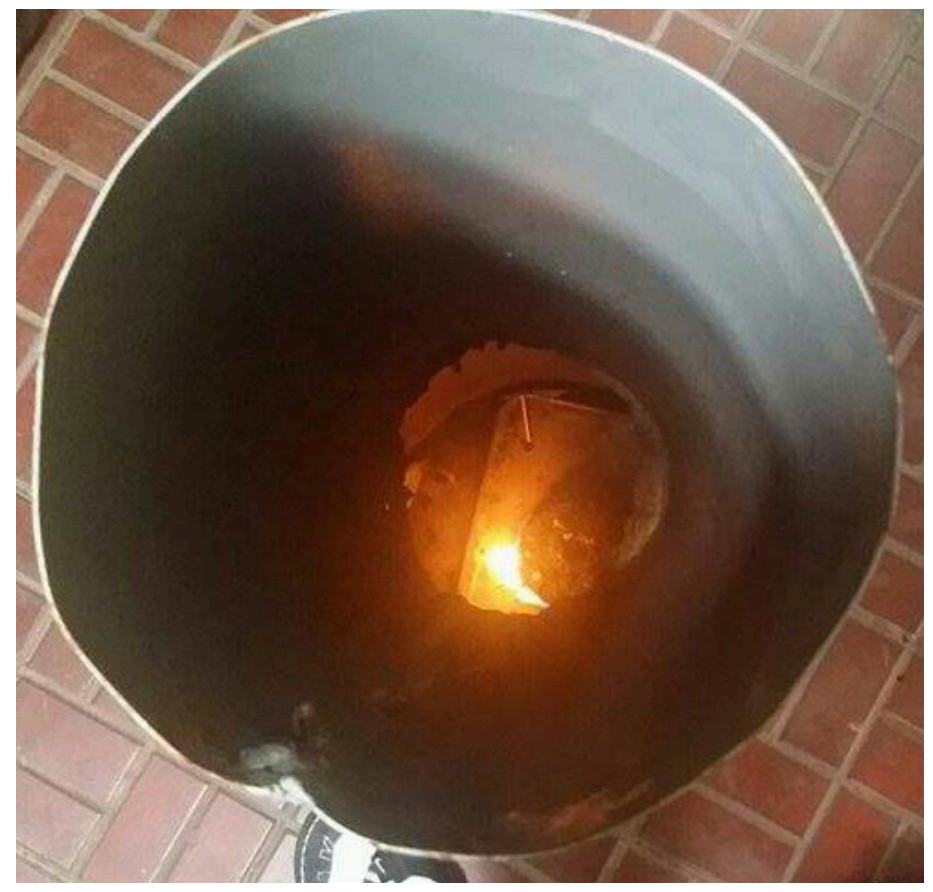

Etapa de evaluación de las briquetas.

La etapa final de nuestra investigación se realizó en un cuarto cerrado para controlar variables que influyen de manera directa en el resultado de datos como lo es el viento. En esta etapa se evaluaron dos parámetros fundamentales planteados en nuestros objetivos: la eficiencia y las emisiones de $\mathrm{CO}$ de las briquetas con diferentes tipos de sustratos forestales.

Las siguientes evaluaciones se realizaron en la Turbo cocina fabricada por estudiantes de ingeniería industrial de Farem Estelí $\mathrm{y}$ en una pequeña parrilla casera.

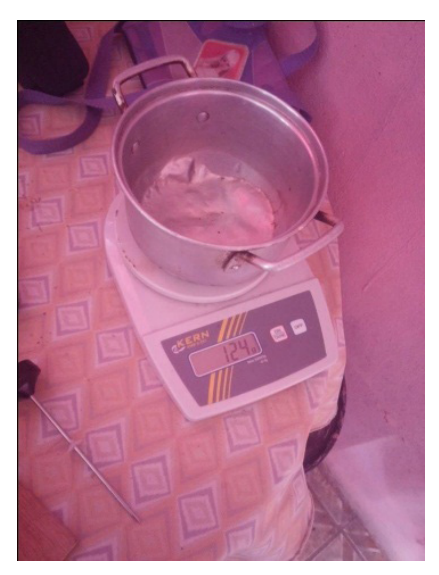

La evaluación de eficiencia se determinó por la prueba de ebullición de agua (WBT por sus siglas en inglés) versión 4.2.2. La cual fue modificada y se realizó la prueba de Arranque en frio (coldstar) en las cuales se evaluaron las siguientes variables:

- Tiempo de ebullición: calculando el tiempo desde que tarda en llegar al punto de ebullición del agua.
- Velocidad de combustión: es la medida del consumo de combustible (leña o briquetas) que se requiere para lograr hervir el agua de un recipiente.

- Eficiencia térmica: es la transferencia de energía del combustible a la olla.

- Consumo especifico del combustible: cantidad de combustible requerida para hacer hervir un litro de agua empezando con la estufa fría,

- Equivalente de combustible consumido: cantidad de combustible que se quemó.

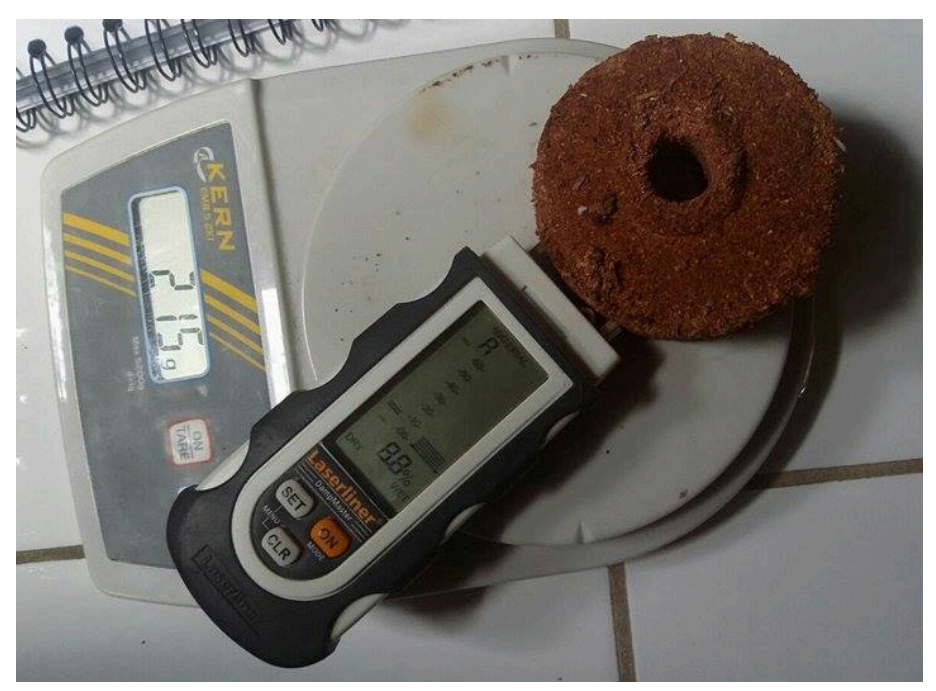

\section{Emisiones de monóxido de carbono (CO)}

Durante la prueba WBT se utilizó una olla de aluminio casera para contener 1 litro de agua y una balanza analítica marca KERN para el pesaje de briquetas, residuos de carbón y ceniza. Se empleó un termómetro digital marca testo, un medidor de humedad de madera, marca Laserliner, y un medidor de monóxido de carbono marca BK PRECISION 627 para evaluar las emisiones de $\mathrm{CO}$. Estos instrumentos digitales fueron proporcionados por el laboratorio de energía renovable de FAREM-ESTELI.

Para la obtención de las variables a evaluar se automatizaron mediante un formato de Excel extraído del protocolo de prueba de ebullición de agua (WBT), mediante el cual se ingresaron valores conocidos para que automáticamente se determinaran de manera eficiente las variables desconocidas. 


\section{RESULTADOS Y DISCUCIÓN}

Esta investigación al ser la primera que se realiza en FAREM-ESTELI con respecto a la evaluación de parámetros físicos y químicos de las briquetas elaboradas de diferentes residuos forestales, radica su importancia en los resultados obtenidos de las diferentes pruebas realizadas para considerar a las briquetas como alternativa para reducir el uso leña como combustible, y aprovechar diferentes residuos que no tienen un tratamiento adecuado en nuestra ciudad.

Evaluar los parámetros físicos y químicos de las briquetas elaboradas con la maquina briquetadora construida en FAREM-Estelí.

En la etapa de pre-evaluación (cuadro 1) se presentan todos los tipos de briquetas que se fabricaron para determinar cuál de ellas pasaba a la etapa de evaluación. Para ello las briquetas debían tener en su prueba física una calificación de excelente y pudiesen encender con facilidad. Esta etapa de evaluación se realizó en condiciones controladas utilizando una cama de madera y ocote para el encendido inicial de las briquetas.

Se presenta el cuadro de resultados para lo cual el papel actuó como aglutinante en la mayoría de casos.

Tabla 1. Resultados de las Pruebas de Preevaluación.

\begin{tabular}{clll}
\hline$N^{\circ}$ & $\begin{array}{r}\text { Tipo de } \\
\text { briqueta }\end{array}$ & $\begin{array}{r}\text { Prueba física } \\
\text { (Exelente/ } \\
\text { Regular/Malo) }\end{array}$ & $\begin{array}{c}\text { Prueba de encendido } \\
\text { (Enciende/No } \\
\text { enciende) }\end{array}$ \\
\hline $1 \quad \begin{array}{l}\text { Colocho de } \\
\text { aserrín } 67 \% \\
+ \text { Papel } 33 \%\end{array}$ & Regular & Enciende \\
2 & $\begin{array}{l}\text { Aserrín } \\
\text { fino } 50 \%+ \\
\text { papel } 25 \%+ \\
\text { cascarilla de } \\
\text { café } 25 \%\end{array}$ & Excelente & Enciende \\
& &
\end{tabular}

3 Granza 50\%

+ Aserrín fino

$25 \%+$ Papel

$12.5 \%+$ flor

de pino $12.5 \%$

4 Pasto seco

$50 \%+$ Papel Regular Enciende

$50 \%$

5 Papel 50\%

+ Aserrín

colocho

$25 \%+$ Ramitas

$10 \%+$ Granza

Excelente Enciende

$8.4+$ flor de

pino $6.6 \%$

6 Papel $25 \%+$

Cascara de

café $25 \%+$

Aserrín fino

Excelente Enciende

$25 \%+$ Aserrín

colocho $25 \%$

7 Aserrín 100\% Malo No enciende

8 Papel $100 \%$ Malo No enciende

9 Aserrín fino

$80 \%+$ papel Excelente Enciende

$20 \%$

10 Ramitas $40 \%$

+ aserrín fino

$40 \%$

Regular No enciende

$1160 \%$ aserrín + $40 \%$ papel

Excelente Enciende

$12 \quad 100 \%$ pasto seco

Regular

Enciende

Las tipos de briquetas que pasaron la etapa de preevaluación según se observó y determino fueron debido a que, estas al ser manipuladas durante su fabricación, transporte y secado conservaron su estructura fuerte, no se partieron ni sufrieron fisuras. Además en la prueba de encendido que consistió en medir la facilidad que tienen las briquetas en encender para lo cual se utilizó una cama de madera u ocote igual para todas las briquetas y se obtuvieron excelentes resultados ya que cuando al quemarse la cama las briquetas estas quedaban encendidas por un tiempo considerable hasta consumirse.

Las tipos de briquetas que pasaron la etapa de preevaluación fueron las siguientes: 
1. Aserrín fino $50 \%+$ papel $25 \%$ + cascarilla de café $25 \%$

2. Granza $50 \%+$ Aserrín fino $25 \%+$ Papel $12.5 \%+$ flor de pino $12.5 \%$

3. Papel $50 \%+$ Aserrín colocho $25 \%+$ Ramitas $10 \%+$ Granza 8.4 + flor de pino $6.6 \%$

4. Papel $25 \%+$ Cascara de café $25 \%+$ Aserrín fino $25 \%+$ Aserrín colocho $25 \%$

5. Aserrín fino $80 \%+$ papel $20 \%$

6. $60 \%$ aserrín $+40 \%$ papel

La etapa de evaluación de las briquetas se realizó bajo condiciones controladas cada una de las pruebas para tener uniformidad en los resultados, una vez encendida la briqueta se procuró no darle aireación, se esperó hasta que volviera a encender por sí sola, observando en algunas que se apagaron completamente en un lapso de tiempo determinado, estas pruebas las realizamos en una parrilla casera debido a que el calor de las briquetas se traslada directamente a la olla generando mayor aumento de temperatura y menor desperdicio de combustible, se controlaba cada una de las pruebas asegurando uniformidad $y$ evitando variaciones externas que influyeran de manera directa. Al realizar las pruebas se ubicó el centro de la parrilla una briqueta la con la cual se pretende alcanzar el punto de ebullición de un litro de agua en un tiempo determinado.

Para el funcionamiento de las briquetas se necesita un fuego inicial de otro combustible en este caso se optó por rociar el orificio central de las briquetas con $5 \mathrm{ml}$ de Diluyente (zener) para provocar el encendido sin afectar mucho la naturalidad de las pruebas.

Se comienzan con las evaluaciones pertinentes para las briquetas que pasaron la pre evaluación realizando de manera modificada la WBT, al mismo tiempo las emisiones de monóxido de carbono ( $\mathrm{CO}$ ). Además se calcula el poder calorífico de los diferentes tipos de briquetas dependiendo sus materiales de elaboración dato que es fundamental para cálculo del WBT en el programa de Excel.
Cabe mencionar que las pruebas se realizaron con leña de pino $(90 \mathrm{~g})$ y así obtener un patrón de comparación necesario a la hora de evaluar las briquetas ya que a este combustible es el que se pretende reducir su uso. Las pruebas se realizaron similarmente a la de las briquetas para tener uniformidad en los datos y no tener alteración en los resultados.

En la metodología se mencionan diferentes variables a evaluar con el WBT que son las siguientes:

Tiempo de ebullición: calculando el tiempo desde que tarda en llegar al punto de ebullición del agua.

El gráfico 1 muestra los resultados obtenidos de esta prueba.

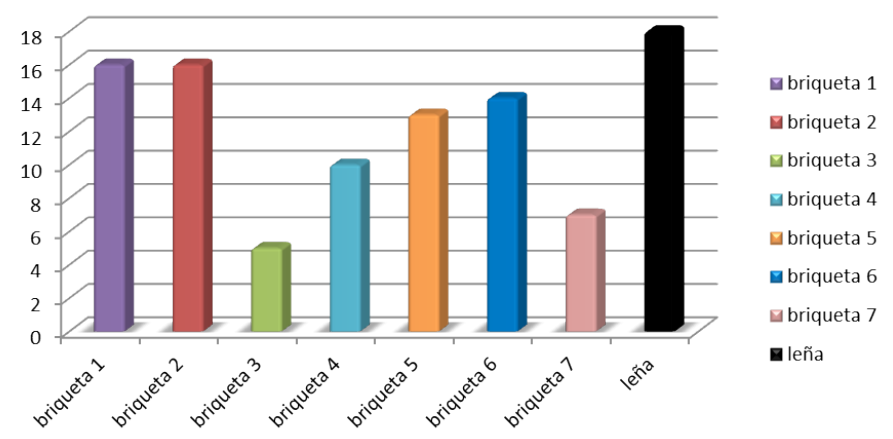

Gráfica 1: Tiempo de ebullicion del agua (minutos)

Como se observa en la gráfica los tiempos son relativamente bajos puesto que ninguna briqueta alcanzo el punto de ebullición debido a que el combustible no se quemaba completamente o que se apagaba rápido debido a que no se utilizó encendido externo de manera adecuada para no alterar las pruebas.

Por tanto este factor no se considera determinante a la hora de determinar cuál briqueta es mejor o no.

Velocidad de combustión: es la medida del consumo de combustible (leña o briquetas) que se requiere para lograr hervir el agua de un recipiente. 


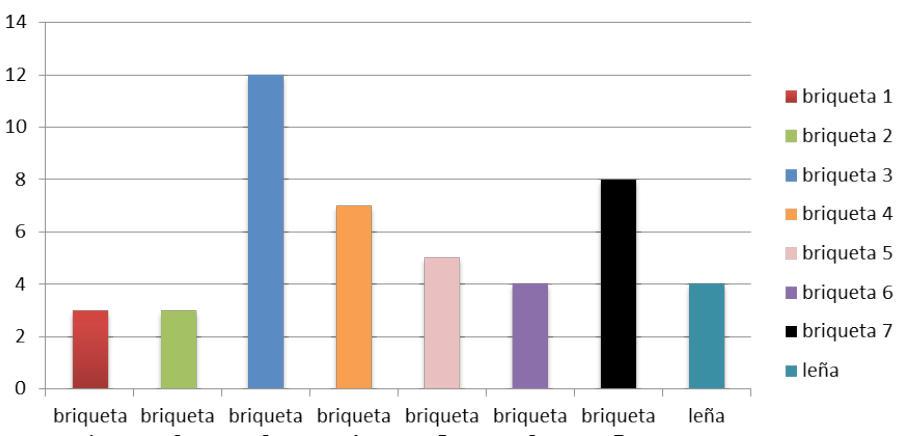

Gráfica 2: Ġraficả de vêlocidăd de ćombưstión expresada en gramos consumidos por minuto

La velocidad de combustión mayor se da en la briqueta 3 (Papel 50\% + Aserrín colocho 25\%+Ramitas 10\%+ Granza 8.4 + flor de pino 6.6\%), como se observa en la gráfica se consume 12 gramos cada minuto transcurrido lo cual nos quiere decir que tiene mayor capacidad de combustión aprovechando a mayor velocidad el contenido del combustible, pero también significa que es mayor la cantidad de combustible utilizado para hacer llegar el agua a su punto de ebullición.

Eficiencia térmica: es la transferencia de energía del combustible a la olla.

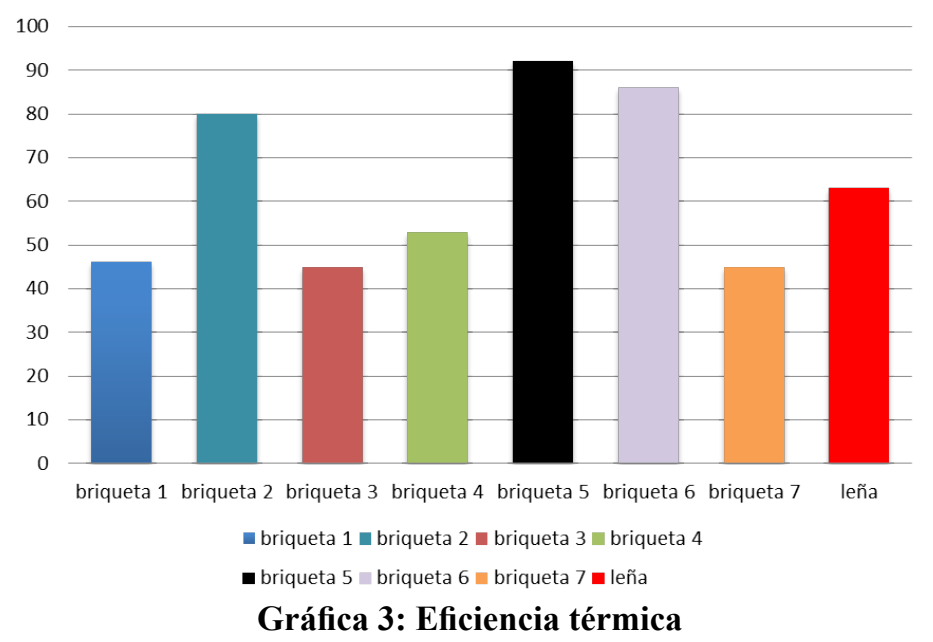

Este es una variable fundamental a la hora de determinar la factibilidad de un combustible. En la gráfica se observa como la mejor eficiencia térmica la presenta la briqueta 5 , con aproximadamente un $92 \%$ siendo la más relevante y la briqueta 6 y 2 con $86 \%$ y $80 \%$ respectivamente. Tanto la leña como las otras briquetas se mantienen en un estándar entre 45 y $63 \%$ lo cual es una eficiencia promedio.
Consumo especifico del combustible: cantidad de combustible requerida para hacer hervir un litro de agua empezando con la estufa fría.

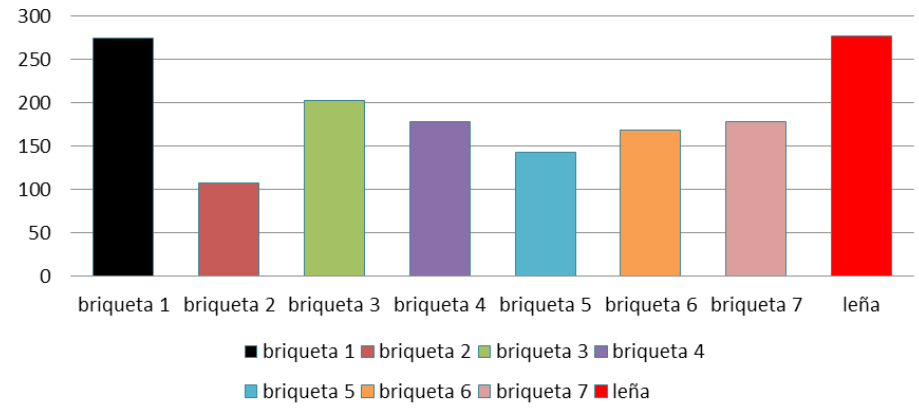

Gráfica 4: Consumo especifico del combustible (g/litro)

En este caso el combustible adecuado para hacer hervir 1 litro de agua la que presenta menor consumo específico es la briqueta 2. La leña y la briqueta 1 presentan un elevado consumo específico para hervir un litro de agua.

Equivalente de combustible consumido: cantidad de combustible que se quemó.

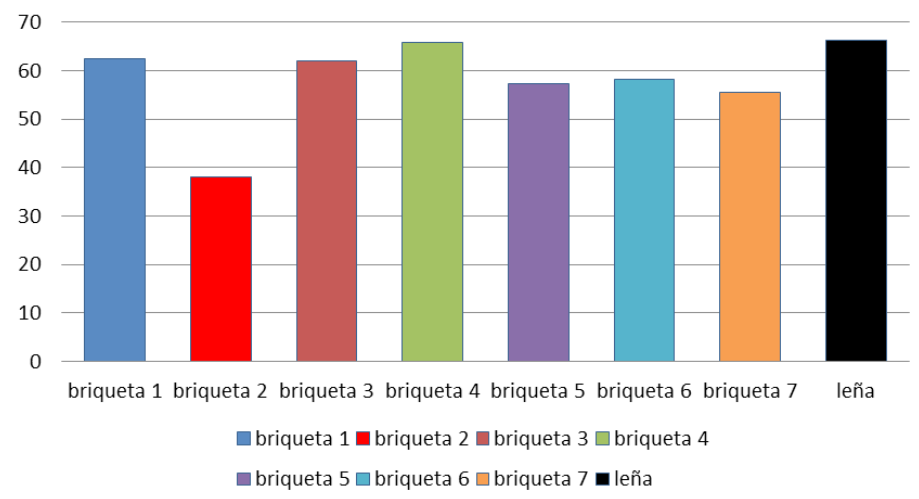

Gráfica 5: Consumo de combustible en gramos

La briqueta 4 y la leña consumen mayor combustible seco lo que quiere decir que aprovechan más y tienen mejor capacidad de combustión respecto a las otras briquetas. La briqueta 2 es la de menor consumo de combustible dejando mayor cantidad de residuos no aprovechados en la combustión.

\section{Emisiones de monóxido de carbono (CO).}

El monóxido de carbón es un gas altamente toxico y peligroso para los seres humanos ya que al entrar 
al torrente sanguíneo reemplaza el oxígeno y pueden morir por intoxicación. Por tanto es una variable fundamental en nuestra evaluación la cual se determina dividiendo las emisiones totales de cada prueba entre el peso del combustible ya sea el de la briqueta y el de la leña.

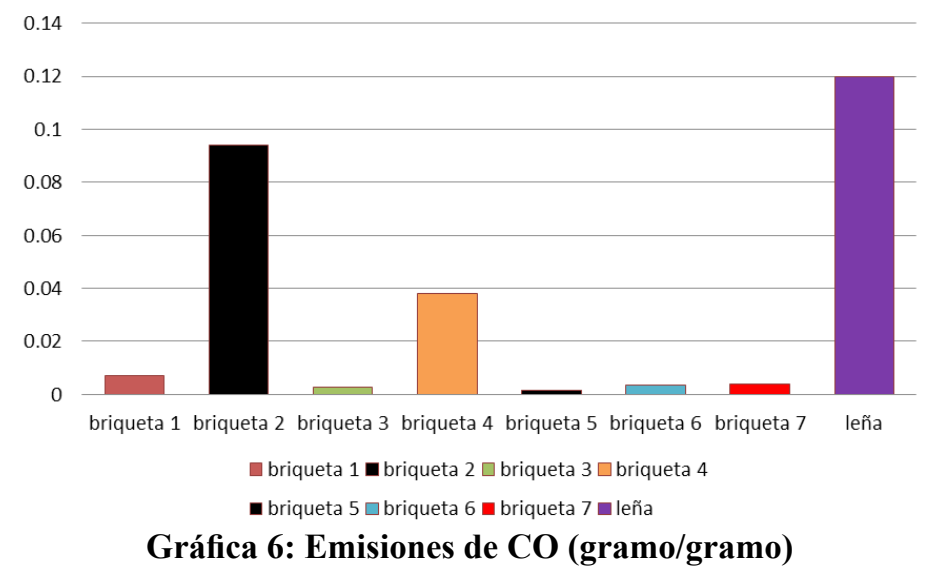

Es notable como la leña es tan destructiva en cuanto a emisiones de $\mathrm{CO}$ se refiere emitiendo 12 gramos de $\mathrm{CO}$ por cada 100 gramos de leña quemados lo cual la deja en desventaja con respecto a las briquetas. La briqueta 5 es la que presenta menores o casi nulas emisiones de $\mathrm{CO}$ con tan solo 0.0015 gramos de $\mathrm{CO}$ por cada 100 gramos haciéndola esta la más conveniente para sustituir la leña como combustible pues genera menos impacto medio ambiental que en comparación a la leña es casi nulo.

\section{CONCLUSIONES}

La elaboración de las briquetas fue exitosa logrando una excelente compactación reduciendo los niveles de humedad y aumentando la densidad del producto. En la fabricación de briquetas el corte y la descomposición parcial son determinantes para una buena estructura.

La etapa de pre-evaluación fue fundamental para determinar cuál briqueta fue conveniente puesto que en algunos casos la briqueta se quebraba por su mala estructura o no quemaba lo que hubiese quitado tiempo en vano a la hora de evaluar.
La leña es un generador de energía que emite cantidades de $\mathrm{CO}$ a la atmosfera exageradas por lo cual es conveniente buscar alternativas para su sustitución como las briquetas.

La briqueta 5 de Granza 50\% + Aserrín fino 25\% + Papel $12.5 \%$ + flor de pino $12.5 \%$ es la mejor opción según la etapa de evaluación siendo esta la que emite menores cantidades de $\mathrm{CO}$ que son casi nulas obteniendo una eficiencia térmica de $92 \%$ puesto que su consumo especifico es bajo con tan solo 143 gramos por litro de agua.

Si es posible sustituir la leña como combustible ya que las briquetas como la briqueta 5 la briqueta 3 cuentan con mayor eficiencia térmica, tienen menor consumo, mayor lapso de combustión y emisiones de CO aceptables.

En la turbo cocina las briquetas cuentan con mayor eficiencia y debido a que el viento es casi nulo su periodo de encendido es alto hasta llegar al punto de consumirse toda, no se pudo evaluar correctamente por la falta de briquetas pues para la turbococina se necesita al menos $2 \mathrm{~kg}$ de briquetas para que ceda calor a la olla pues queda muy alejada la cámara de combustión.

No todos los residuos tienen la capacidad de usarse para la fabricación de briquetas. Sin embargo no se descarta que al estar mezclado con otros materiales tengan mayor eficiencia. Se tienen que evaluar previamente al hacer cualquier recomendación para el uso.

Este estudio ayudó a tener una perspectiva amplia de lo que puede ser el uso de residuos agrícolas para la fabricación de briquetas, y más adelante mediante tecnologías avanzadas se puedan desarrollar de una manera eficiente. Así mismo puede ser un pilar para futuras proyecciones que se realicen a pequeña y gran escala, en las diferentes industrias de nuestra ciudad. 


\section{RECOMENDACIONES}

- Realizar más evaluaciones de briquetas con los protocolos WBT bajo condiciones similares y diferentes de viento, para contar con una base de datos para estudios posteriores.

- Diseñar una estufa a medida y según las características de encendido de las briquetas, para evaluar y comparar con los resultados ya existentes.

- Recolectar los residuos agrícolas después que estos hayan estado expuestos al aire libre por largo tiempo para que la descomposición parcial esté avanzada y así acortar los tiempos de descomposición y secado durante la fabricación de las briquetas.

\section{BIBLIOGRAFÍA}

Comisión Forestal para América Latina y el Caribe FAO. (1999). Situación Forestal de América Latina y el Caribe. Obtenido de http://www.fao. org/docrep/meeting/x6004s.htm.

Rivas, A. (2001). Estudio de la valoracion energetica de los combustibles forestales. Santiago de Compostela. Mexico

Morini, S. (2011). Perdidas y Desperdicios de Recursos Forestales en el Mundo. Düsseldorf, Alemania

Ortiz, L. (2003). La Biomasa como fuente de energía. Madrid.

Barrera, J. (2008). Briquetas, la mejor forma de reutilizar los residuos forestales. Madrid

Kollman, F. (2007). Tecnología de la madera y sus aplicaciones. Madrid 